\title{
Effects of antibiotics on acquired immunity in vivo - current state of knowledge
}

\author{
M. Pomorska-Mól, Z. Pejsak \\ Department of Swine Diseases, National Veterinary Research Institute, \\ Al. Partyzantów 57, 24-100 Puławy, Poland
}

\begin{abstract}
Antibiotics are widely used in the therapy of infections. Besides the respective interactions between antibiotics and pathogens it seems that antibiotics also directly interact with the immune system. Some commonly used antibiotics are currently known to have effects on the innate immune response, as shown by in vitro, ex vivo and also in vivo animal experiments and clinical studies. Most of the experimental papers published to date, as well as most reviews, relate to how antibiotics affect the innate immune response or non-specific monocyte or lymphocyte proliferation. However the effects of antibiotics on the adaptive immune response are still not well characterized. This review of the literature considering different in vivo experiments indicate the real importance of interrelations existing between acquired immune responses and antibiotics, however, the mechanism of immunomodulatory effects of antibiotics are still poorly understood. Currently, data on the immunomodulating effects of antibiotics often remain heterogeneous, contradictory or insufficient, but most results published to date revealed the immunosuppressive effect of antibiotics on the antigen-specific immune response in vivo. In pigs as well as in poultry herds, it is not uncommon practice to add antibiotics to drinking water or feed at the time of vaccination. Information on the effects of such practices on the immune system of animals is restricted and more in vivo studies are needed to investigate the effects of antimicrobial drugs on the immune system, especially in the field conditions.
\end{abstract}

Key words: antibiotics, immunomodulation, acquired immunity, in vivo

Abbreviations: CMI- cell mediated immunity, IFN- $\gamma$ - interferon gamma, IL- interleukin, i.p. - intraperitoneally, ND- Newcastle disease, NVD - Newcastle disease virus, SRBCs - sheep red blood cells, s.c. - subcutaneously

\section{Introduction}

Antibiotics are widely used as bacteriostatic or bactericidal drugs in the therapy of infections. Besides the respective interactions between antibiotics and pathogens, and between the immune system and pathogens, it seems that antibiotics also directly interact with the immune system. Results from last years have shown that many antibiotics - in addition to their antibacterial activity - have also immunomodulatory properties and can induce a significant modulation of the immunity (Skopińska-Różewska et al. 1981, Yamamoto et al. 2000, Munir et al. 2007, Brisbin et al. 2008, Tauber and Nau 2008, Khalifeh et al. 2009, Radomska-Leśniewska et al. 2010a, Radomska-Leśniewska et al. 2010b). Chemotherapy of bacterial 
infections can no longer be regarded as an interaction between drugs and bacteria. Undoubtedly the immune system of the host is also strongly involved in this interaction. Some commonly used antibiotics are currently known to have effects on the innate immune response. Concerning macrophage-monocyte functions, in vitro studies have shown that macrolides stimulate phagocytic chemotaxis (Carlone et al. 1989), promote monocyte-macrophage differentiation (Keiho et al. 1994, Xu et al. 1996, Yoshida et al. 2005), and increase the killing capacity of macrophages (Cuffini et al. 1989). Tetracyclines inhibit phagocytic chemotaxis and granuloma formation (Webster et al. 1994). As for cytokines, tetracyclines inhibit interleukin-1 (IL-1) and tumor necrosis factor alfa (TNF-alfa) production by human macrophages (Shapira et al. 1996, Milano et al. 1997) while macrolides inhibit IL-1 production by murine peritoneal macrophages (Takeshita et al. 1989, Morikawa et al. 1994). In regard to lymphocytes, macrolides suppress lymphocyte proliferation and the proliferative response to human peripheral blood mononuclear cells stimulated by polyclonal T-cell mitogens (Morikawa et al. 1994).

An in vitro study carried-out on chicken-derived phagocytic cells showed that tilmicosin is concentrated in phagocytes due to their lipophilic properties (Chin et al. 2000). The uptake of this antibiotics resulted in an increase in lysosomal enzyme activity, which suggest a complex interaction between phagocytic cells and tilmicosin (Chin et al. 2000). Florfenicol inhibited in vitro phagocytosis activity of neutrophils in bovine (Paape et al. 1990).

Most of the experimental papers published to date, as well as most reviews, relate to how antibiotics affect the innate immune response or non-specific monocyte or lymphocyte proliferation (Carlone et al. 1989, Keiho et al. 1994, Xu et al. 1996, Labro 2000, Yoshida et al. 2005). However, the effects of antibiotics on the adaptive immune response in vivo are still not well characterized. The knowledge of possible influences of antibiotics on the immune response seems to be of great importance for the clinical approach to the process of therapy and/or vaccination.

Here, recently published studies on immunomodulatory properties of antibiotics with regard to antigen-specific immune response in vivo are reviewed and disscused.

\section{Influence of antibiotics on acquired immunity in vivo}

There is limited information on how antibiotics affect the effector arms of adaptive immunity - speci- fic-antigen induced antibody production and how they influence the specific-lymphocyte proliferation to date. Moreover, the results of previous studies have been conflicting and depended on the type and dose of the antibiotic, as well as on the route of administration and duration of treatment.

Khalifeh et al. (2009) investigated the effects of antibiotic treatment at the time of chicks vaccination against Newcastle disease (ND) on the immune response after Newcastle disease virus (NDV) exposure. They demonstrated that tilmicosin, florfenicol and enrofloxacin clearly targeted the humoral immune response and resulted in a decrease in antibody production. In addition, oral antibiotic treatment, probably through maintaining the intact mucosa surfaces and changing the normal flora bacterial content, caused an upregulation in the threshold of the cell mediated immunity (CMI) (Khalifeh et al. 2009).

Beneficial effects of salinomycin and monensin were found on CMI responses after vaccination of broilers against NDV, whereas another study reported an immunosuppressive effects of salinomycin on the immune response generated against NDV (Shalaby et al. 1993, Munir et al. 2007).

Roszkowski et al. (1985) evaluated the effects of beta-lactams and clindamycin on immunological parameters in the mice-model. They have shown that mezocillin and piperacyllin inhibited both the cellular and humoral responses. Moreover, both drugs impaired the proliferative activity of lymphocytes. Suppression of the cellular and humoral immunity was also observed after 7 days treatment with cefotaxime and amikacin. However, the degree of inhibition was not as strong after the application of mezlocillin and piperacyllin. Moreover, the normalization of the immune reactivity was faster.

Effect of clindamycin and lincomycin on angiogenic activity (angiogenesis is regarded as a part of the cell immunity) was studied also by Radomska-Leśniewska et al. (2010b). The influence of these antibiotics on neovasuclar reaction induced in $\mathrm{BALB} / \mathrm{c}$ mice skin by mononuclear cells were evaluated. Mice received antibiotics subcutaneously (s.c.) over 3 days at doses 3, 15 or $75 \mathrm{mg} / \mathrm{kg}$. Mice from the control group were injected with PBS. This study has shown that clindamycin stimulated angiogenic response at all administered doses, while lincomycin did not alter neovascular response. Stimulating effects on the angiogenic activity of human blood mononuclear cells were also found by the same authors with regard to selected cephalosporines (cefuroxime and ceftazidime) while the others (cefradine, ceftriaxone, cefsulodin) inhibited angiogenic response (Radomska-Leśniewska et al. 2010a). 
Demkow et al. (1998) evaluated the influence of rifampicin on some parameters of acquired immunity. This study has shown that rifampicin administered at dose of $50 \mathrm{mg} / \mathrm{kg}$ to B6AF1 mice slightly stimulated the production of anti-SRBC antibodies and suppressed the cellular response in vivo. Additionally this drug inhibited angiogenesis induced by leukocytes.

Borowski et al. (1985) investigated the effect of cefotaxime and cephradine on the humoral and cellular immune response in BALB/c $\left(\mathrm{H}-2^{\mathrm{d}}\right)$ mice. They found that cefotaxime at therapeutic doses did not affect the humoral and cellular immune response against sheep erythrocytes, whereas cephradine suppressed the humoral response at doses corresponding to those used for the treatment of patients.

In the study conducted by Bellahsene et al. (1985) the influence of doxycycline on cell-mediated immunity as well as on humoral response in CBA/H $\left(\mathrm{H}-2^{\mathrm{k}}\right)$ mice were evaluated. Every morning, the animals were given doxycycline intraperitoneally at doses of $2.5 \mathrm{mg} / \mathrm{kg}$ (human therapeutic dose). Both agglutinating and hemolytic antibody response to sheep erythrocytes was slightly but significantly decreased after treatment with doxycycline, though there was no inhibition of splenic production of antibodies to sheep erythrocytes (as measured by the number of plaques of hemolysis detected). The results indicate that the immune response in mice can be moderately inhibited by doxycycline.

The immunomodulatory action of ampicillin has been shown by Skopińska-Różewska et al. (1985) and Włodarska et al. (1987). In the study performed by Skopińska-Różewska et al. (1985) ampicillin was injected s.c. at daily doses of $250 \mathrm{mg} / \mathrm{kg}$ for 7 days. On the day following the last injection, the mice received a single injection of SRBC $\left(2 \times 10^{8}\right.$ cells/mouse). The anti-SRBC antibodies in mice serum were determined 7 days later by direct haemagglutination test. In this study, ampicillin was found to have an enhancing effect on the expression of FcM human lymphocyte receptors, on the angiogenic potential of human lymphocytes and on the production of anti-SRBC antibodies after in vivo administration to low-responders strains of mice (various strains). Włodarska et al. (1987) evaluated the effect of ampicillin given to pregnant $\mathrm{C} 3 \mathrm{HW}$ mice on the immune reactivity in their offspring. Prospective mothers were injected with 10 $\mathrm{mg}$ of ampicillin daily, during last 8-9 days of pregnancy. The lowered cellular immunity of the progeny and reduced percentage of $\mathrm{T}$ lymphocytes in the lymph nodes were found in mice born to ampicillin-treated mothers, while the humoral immunity was enhanced in the same animals.

Woo et al. (1999) investigated whereas clatithromycin, doxycycline or ampicillin modulate vac- cine-induced humoral immune response in BALB/c $\left(\mathrm{H}-2^{\mathrm{d}}\right)$ mice after intraperitoneal administration from one day prior immunization to day 27 post immunization. As antigens series of common vaccines were used (tetanus toxoid, pneumococcal polysaccharide vaccine, hepatitis B virus vaccine, live attenuated $S$. typhi transformed with pBR322). The classic primary antibody responses induced by tetanus toxoid, pneumococcal polysaccharide vaccine and hepatitis B virus vaccine were suppressed by clarithromycin and doxycycline (IgM concentration were significantly lower than those in the ampicillin and control groups). The authors have speculated that this is partly due to a suppression of the T- and B-cell interaction in the production of antibodies (Woo et al. 1999). As it has been shown previously (Morikawa et al. 1994), clarithromycin and doxycycline can inhibit production of some interleukin (IL) by T lymphocytes in vitro. However, this cannot fully explain the suppression, since antibody production to T-cell independent antigen (pneumococcal polysaccharide vaccine) was also restricted. Additional explanation presented by the authors is that other possible targets of action of antibiotics investigated may include antigen presentation or co-stimulatory signals (Woo et al. 1999).

As it has also been shown by Woo et al. (1999), clarithromycin suppressed additionally the humoral response against hepatitis $\mathrm{B}$ antigen with regard to IgG1 class of antibodies. It would be interesting to know whether clarithromycin has the same effect on immunization in humans vaccinated against hepatitis B. No effect on IgG1 was found with respect to others vaccines tested by Woo et al. (1999). In contrast, after vaccination of mice with live attenuated $S$. typhi, the levels of specific antibodies was higher in animals receiving ampicillin, clarithromycin and doxycycline. It seems that the influence of antibiotic on the humoral immune response was dependent mainly on the properties of antigens (Woo et al. 1999). In other study by Woo et al. (2000) the vaccine against S. typhi was given orally. This study has shown that ampicillin improves the B-cell response and the antigen-specific T-cell response after immunization. The mechanism of this enhance is probably by giving $S$. typhi deriving from vaccine a survival advantage against the normal bacterial microflora of the intestine. Woo et al. (2000) revealed that ampicillin significantly suppressed the normal flora of the gut, resulting in a relatively higher rate of recovery of $S$. typhi from the feces. These observations have important implications for vaccinations (Woo et al. 2000). These findings might be useful in selective prophylactic vaccinations programs, such as vaccination nonresponders to routine immunization. However, with concerns about the increasing rate of antibiotic resistance worldwide, the incorpor- 
ation of an antibiotic as a part of routine vaccination schedule could be problematic.

Jaménez-Valera et al. (1995) found that ciprofloxacin injected for 3 days to BALB/c (H-2 ${ }^{\mathrm{d}}$ mice suppressed the indirect ( $\operatorname{IgG}$ ) plaque forming cell response to SRBCs, whereas the response of IgM-forming cells remained unchanged. The dosages used in this study were similar to those used in human therapy. When 3-day treatment was started at $24 \mathrm{~h}$ after immunization with sheep erythrocytes, the response of IgM-forming cells was increased, whereas the response of IgG-forming cells was suppressed. Delayed-type hypersensitivity to sheep erythrocytes was significantly suppressed in animals that received ciprofloxacin for 3 days and were immunized subcutaneously $24 \mathrm{~h}$ after the last injection. Leukopenia and increase in the level of granulocyte-macrophage colony-forming cells in the bone marrow were also observed in ciprofloxacin-treated mice.

Williams et al. (2005) found that moxifloxacin and ciprofloxacin had pronounced effects on both Th1 and Th2 cytokine expression, without altering Th1/Th2 ratios, while clarithromycin decreased only IL-4 expression so that the Th1/Th2 ratio increased. $\mathrm{T}$ helper lymphocytes are important for both cell-mediated and humoral immunity. The particular type of the immune response is determined by the differentiation of precursor najve $\mathrm{T}$ helper (Th0) cells into Th1 or Th2 cells; a process is dependent upon local cytokine concentrations, antigen load and mode of antigen presentation. Each cell subset secretes a particular array of cytokines which further augment the differentiation into that subset. Th1 lymphocytes produce mainly IFN- $\gamma$ and favor cell-mediated immunity, while Th2 lymphocytes secrete predominantly IL-4 and favor humoral immunity (Williams et al. 2005). Since a Th1 profile is considered favorable for resolution of infection, elucidation of immunomodulatory profiles of antibiotics may permit more rational antibiotic choice in future (Williams et al. 2005)

Effects of cefodizime and cefotaxime on the cellular and humoral immune responses were investigated by Pulverer (1992) on mice model. Antibiotics were injected subcutaneously into BALB/c $\left(\mathrm{H}-2^{\mathrm{d}}\right)$ mice at $10 \mathrm{~h}$ intervals for seven consecutive days at various doses (0.4, 0.8 and $1.2 \mathrm{mg} / \mathrm{day})$. The dosages were calculated according to those used in human medicine. Mice were immunized intraperitoneally (i.p.) with $2 \times 10^{8}$ sheep red blood cells (SRBC). The level of IgM and IgG were evaluated 4 and 7 days after immunization, respectively. For analysis of $\mathrm{T}$ cell proliferation, the lymphocyte proliferation assay and delayed type hypersensitivity were used. Cefodizime, in contrast to cefotaxime, did not influence the production of immunoglobulins ( $\mathrm{IgM}$ and $\mathrm{IgG}$ ) or the delayed type hypersensitivity, while cefotaxime was responsible for a remarkable and long-lasting inhibition of the IgM production. The same trends could be seen concerning the $\operatorname{IgG}$ antibody response. Cefotaxime at the dose of 1.2 $\mathrm{mg} /$ day/ BALB/c (H-2 ${ }^{\mathrm{d}}$ mouse over seven days was responsible for a significant inhibition of lymphocyte proliferation (Pulverer 1992).

Ortega et al. (2000) investigated the effect of aztreonam, a monobactam antibiotic, on different parameters of acquired immunity in BALB/c $\left(\mathrm{H}-2^{\mathrm{d}}\right)$ mice. It was found that various doses of this antibiotics i.p. injected into $\mathrm{BALB} / \mathrm{c}\left(\mathrm{H}-2^{\mathrm{d}}\right)$ mice induced an increase in the lymphoproliferative response to specific mitogens and in the production of IL-2 by splenic cells, as well as a decrease in the response of this immune population to sheep erythrocytes, lower total blood cell counts, and lower percentage of monocytes than that determined in untreated $\mathrm{BALB} / \mathrm{c}\left(\mathrm{H}-2^{\mathrm{d}}\right)$ mice. The increase in the lymphoproliferative response detected in $\mathrm{BALB} / \mathrm{c}\left(\mathrm{H}-2^{\mathrm{d}}\right)$ mice treated with aztreonam could be related to the enhancement of IL-2 production by splenic cells. Interleukin-2 is known to increase lymphocyte proliferation. These results point to a modulatory effects of aztreonam on the immune response in mice and could be of interest in antibacterial therapy with this drug.

\section{Conclusion}

This review of the literature considering different in vivo experiments reveals the real importance of the interrelations existing between the specific immune responses and the antibiotics, however, the mechanism of immunomodulatory effects of antibiotics are still poorly understood. Because there is a wide use of antibiotics either to treat or prevent (metaprophylaxis) bacterial diseases in animals, further studies are necessary to see if these immunomodulatory effects of some antibiotics are clinically relevant in veterinary medicine. Currently, data on the immunomodulating effects of antibiotics often remain heterogeneous, contradictory or insufficient, however, most contributions have revealed the immunosuppressive effect of antibiotics on the antigen-specific immune response in vivo. Thus, for the adequate prescription of antibiotics, their immunomodulating profile should be considered, in the same way as is already the case for the pharmacokinetic profile and the antimicrobial spectrum (Ringoir 1992). In pigs as well as in poultry herds, it is not uncommon practice to add antibiotics to drinking water or feed at the time of vaccination. Information on the effects of such practi- 
ces on the immune system of animals is restricted and more in vivo studies are needed in target animals to investigate the effects of antimicrobial drugs on the immune system and immune response, especially in the field conditions.

\section{References}

Bellahsene A, Forsgren A (1985) Effect of doxycycline on immune response in mice. Infect Immun 48: 556-559.

Borowski J, Jakoniuk P, Talarczyk J (1985) The influence of some cephalosporins on immunological response. Drugs Exp Clin Res 11: 83-88.

Brisbin JT, Gong J, Lusty CA, Sabour P, Sanei B, Han Y, Shewen PE, Sharif S (2008) Influence of in-feed virginiamycin on the systemic and mucosal antibody response of chickens. Poult Sci 87: 1995-1999.

Carlone NA, Cufini AM, Tullio V, Sassella D (1989) Comparative effects of roxithromycin and erythromycin on cellular immune functions in vitro. 2. Chemotaxis and phagocytosis of 3H-Staphyloococcus aureus by human macrophages. Microbios 58: 17-25.

Chin AC, Lee WD, Murrin KA, Morck DW, Merrill JK, Dick P, Buret AG (2000) Tilmicosin induces apoptosis in bovine peripheral neutrophils in the presence or in the absence of Pasteurella haemolytica and promotes neutrophil phagocytosis by macrophages. Antimicrob Agents Chemother 44: 2465-2470.

Cuffini AM, Carlone NA, Tullio V, Borsotto M (1989) Comparative effects of roxithromycin and erythromycin on cellular immune functions in vitro. 3. Killing of intracellular Staphyloococcus aureus by human macrophages. Microbios 58: 27-33.

Demkow U, Radomska D, Chrostowska-Wynimko J, Skopińska-Różewska E (1998) The influence of rifampicin on selected parameters of immunologic response. Pneumonol Alergol Pol 66: 45-53

Jiménez-Valera M, Sampedro A, Moreno E, Ruiz-Bravo A (1995) Modification of immune response in mice by ciprofloxacin. Antimicrob Agents Chemother 39: $150-154$

Keicho N, Kudoh S, Yotsumoto H, Akagawa KS (1994) Erythromycin promotes monocyte to macrophage differentiation. J Antibiot (Tokyo) 47: 80-89.

Khalifeh MS, Amawi MM, Abu-Basha EA, Yonis IB (2009) Assessment of humoral and cellular-mediated immune response in chickens treated with tilmicosin, florfenicol, or enrofloxacin at the time of Newcastle disease vaccination. Poult Sci 88: 2118-2124.

Labro MT (2000) Interference of antibacterial agents with phagocyte functions: immunomodulation or "immuno-fairy tales"? Clin Microbiol Rev 13: 615-650.

Milano S, Arcoleo F, D'Agostino P, Cillari E (1997) Intraperitoneal injection of tetracyclines protects mice from lethal endotoxemia downregulating inducible nitric oxide synthase in various organs and cytokine and nitrate secretion in blood. Antimicrob Agents Chemother 41: 117-121.

Morikawa K, Oseko F, Morikawa S, Iwamoto K (1994) Immunomodulatory effects of three macrolides, midecamycin acetate, josamycin, and clarithromycin, on human T-lymphocyte function in vitro. Antimicrob Agents Chemother 38: 2643-2647.

Ortega E, de Pablo MA, Gaforio JJ, Gallego AM, Alvarez C, Ruiz-Bravo A, de Cienfuegos GA (2000) Modification of acquired immunity in BALB/c mice by aztreonam. Int J Antimicrob Agents 15: 193-199.

Paape MJ, Miller RH, Ziv G (1990) Effects of florfenicol, chloramphenicol and thiamphenicol on phagocytosis, chemiluminescence and morphology of bovine polymorphonuclear neutrophil leukocytes. J Dairy Sci 73: 1734$-1744$.

Pulverer G (1992) Effects of cefodizime and cefotaxime on cellular and humoral immune responses. Infection 20: S41-44.

Radomska-Leśniewska DM, Skopińska-Różewska E，Jankowska-Steifer E (2010a) The effect of selected cephalosporins on angiogenic activity of human blood mononuclear cells. Centr Eur J Immunol 35: 14-19.

Radomska-Leśniewska DM, Skopińska-Różewska E, Malejczyk J (2010b) The effect of clindamycin and lincomycin on angiogenic activity of human blood mononuclear cells. Centr Eur J Immunol 35: 217-222.

Ringoir S (1992) The "infection equation". Infection 20: S75-77.

Roszkowski W, Ko HL, Roszkowski K, Jeljaszewicz J, Pulverer $G$ (1985) Antibiotics and immunomodulation: Effects of cefotaxime, amikacin, mezlocillin, piperacillin and clindamycin. Med Microbiol Immunol 173: 279-289.

Shapira L, Soskolne WA, Houri Y, Barak V, Halabi A, Stabholz A (1996) Protection against endotoxic shock and lipopolysaccharide-induced local inflammation by tetracycline: correlation with inhibition of cytokine secretion. Infect Immun 64: 825-828.

Skopińska-Różewska E, Kamiński M, Nowaczyk M, Moscicka-Wesołowska M, Majewski S, Pazdur J, Malejczyk M (1985) Immunomodulatory action of ampicillin. Folia Biol (Praha) 31: 200-212.

Skopińska-Różewska E, Nowaczyk M, Lao M, Górski AJ (1981) The effect of ampicillin on the expression of Fc-IgG receptors on human peripheral blood lymphocytes. Biomedicine 35: 41-42.

Takeshita K, Yamagishi I, Harada M, Otomo S, Nakagawa $\mathrm{T}$, Mizushima Y (1989) Immunological and anti-inflammatory effects of claritromycin: inhibition of interleukin 1 production of murine peritoneal macrophages. Drugs Exp Clin Res 15: 527-533.

Webster GF, Toso SM, Hegemann L (1994) Inhibition of a model of in vitro granuloma formation by tetracyclines and ciprofloxacin. Involvement of protein kinase C. Arch Dermatol 130: 748-752.

Woo PC, Chow LW, Ma ES, Yuen KY (1999) Clarithromycin attenuates the inflammatory response induced by surgical trauma in a guinea pig model. Pharmacol Res 39: 49-54

Woo PC, Tsoi HW, Leung HC, Wong LP, Wong SS, Chan E, Yuen KY (2000) Enhancement by ampicillin of antibody responses induced by a protein antigen and a DNA vaccine carried by live-attenuated Salmonella enterica serovar Typhi. Clin Diagn Lab Immunol 7: 596-599.

Woo PC, Tsoi HW, Wong LP, Leung HC, Yuen KY (1999) Antibiotics modulate vaccine-induced humoral immune response. Clin Diagn Lab Immunol 6: 832-837.

Williams AC, Galley HF, Watt AM, Webster NR (2005) Differential effects of three antibiotics on $\mathrm{T}$ helper cell 
cytokine expression. J Antimicrob Chemother 56: 502-506 .

Włodarska B, Bany J, Marczak M, Korczak-Komorowski A, Polakowski IJ, Pazdur J, Skopińska-Różewska E (1987) Altered immune reactivity of mice born from mothers treated with ampicillin during gestation. Folia Biol (Praha) 33: 210-215.

Xu G, Fujita J, Negayama K, Yuube K, Hojo S, Yamaji Y, Kawanishi K, Takahara J (1996) Effect of macrolide antibiotics on macrophage functions. Microbiol Immunol 40: 473-479.
Yamamoto K, Takagi M, Endoh YS, Kijima M, Takahashi $\mathrm{T}$ (2000) Influence of antibiotics used as feed additives on the immune effect of erysipelas live vaccine in swine. J Vet Med B Infect Dis Vet Public Health 47: 453-460.

Yoshida K, Sunazuka T, Nagai K, Sugawara A, Cho A, Nagamitsu T, Harigaya Y, Otoguro K, Akagawa KS, Omura S (2005) Macrolides with promotive activity of monocyte to macrophage differentiation. J Antibiot (Tokyo) 58: 79-81. 